JESSIE FRAZELLE

\title{
Out-of-this-World Additive Manufacturing
}

FROM

THINGAMABOBS

TO ROCKETS, 3D

PRINTING TAKES

MANY FORMS.

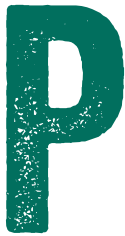

opular culture uses the term 3D printing as a synonym for additive manufacturing processes. In 2010, the ASTM (American Society for Testing and Materials) group, ASTM F42 - Additive Manufacturing, came up with a set of standards to classify additive manufacturing processes into seven categories. Each process uses different materials and machine technology, which affects the use cases and applications, as well as the economics. I went down a rabbit hole researching the various processes in my hunt to buy the best 3D printer lyou can read my reviews on my blog, jessfraz.com]. In this article I will share a bit of what I learned about each process, as well as some of the more interesting use cases I found along the way.

Additive manufacturing has a variety of use cases ranging from thingamabobs to jewelry to metal parts for complex systems to even immense, exciting things like building a boat $^{7}$ or rockets to go into space. Yes, you read that right, both Relativity Space (relativityspace.com) and Launcher Space are using additive manufacturing to build rockets that launch satellites (or other cargo) into space. Relativity Space even built its own additive manufacturing machine, named Stargate, for this purpose, while Launcher partnered with Additive Manufacturing Customized Machines 
(amcm.com) on its engine, E-2 [launcherspace.com].

It's amazing to see the scale and variety of different use cases for additive manufacturing processes. By using additive manufacturing, companies and individuals can go from idea to creation faster than having to involve a thirdparty manufacturing partner. The differentiation in all the different processes enables choosing a process that works best with what you intend to create. Let's continue to dive into each process!

\section{MATERIAL EXTRUSION}

Material extrusion is the most commonly available and least expensive type of 3D printing technology. It represents the largest installed base of 3D printers globally. In material extrusion an object is built by melting and extruding a thermoplastic polymer filament in a predetermined path, layer by layer. Imagine building an object using only a tube of toothpaste. You would slowly build the walls of the object by putting layers of toothpaste on top of each other. Material extrusion works in a similar way.

The most common applications for material extrusion are electrical housings, form and fit testings, jigs and fixtures, and investment casting patterns. The technology commonly used for material extrusion is known as fused deposition modeling, or FDM (lboro.ac.uk).

\section{Fused deposition modeling}

FDM, also known as FFF (fused filament fabrication), works with a range of standard thermoplastic filaments, such as ABS [acrylonitrile butadiene styrene), PLA (polylactic acid), 
PET (polyethylene terephthalate), TPU (thermoplastic polyurethane), nylon, and their various blends.

FDM works in the following way:

1. First, a spool of thermoplastic filament is loaded into the printer. Once the nozzle has heated to the correct temperature, the filament is fed to the extrusion head and into the nozzle, where it melts.

2. Then the extrusion head is connected to a threeaxis system that allows it to move in the $X, Y$, and $Z$ dimensions. The melted material is extruded in thin strands and deposited layer by layer in predetermined locations, where it cools and solidifies. The cooling process can be accelerated by using fans attached to the extrusion head if the device supports it.

3. Filling an area requires multiple passes, similar to coloring with a marker. When a layer is complete, the build platform moves down or the extrusion head moves up, depending on the device, and a new layer is deposited. This process is repeated until the object is completed.

This process tends to result in FDM objects having visible layer lines, unless smoothed, possibly showing inaccuracies around complex features. The toothpaste analogy holds true here, as it would have visible layer lines as well.

While FDM is traditionally used for plastics, Markforged (markforged.com) uses a combination of FDM and MIM (metal injection molding) for its Metal X printers. These machines can print metal and carbon-fiber parts. They use two filament materials-a bound metal powder filament and a ceramic release material-to create the part in a material extrusion process. The part then gets washed to 
break down the polymer binding material. Finally, the part is transformed from a lightly bound metal powder part to a full metal part via sintering. Each step uses a different machine, and the parts require supports, structures that hold the parts and provide strength and resistance against forces like gravity for the object being printed. This demonstrates that you are not tied to a given process but can use aspects of each of these processes to fulfill a given use case. Markforged claims its process is safer and more cost efficient than using a loose metal powder (markforged.com).

\section{VAT PHOTOPOLYMERIZATION}

Photopolymerization is a common approach used by additive technologies to build an object one layer at a time. It occurs when a photopolymer resin is exposed to light of a specific wavelength, causing a chemical reaction that makes it turn solid. Vat refers to inducing this chemical reaction repeatedly in a vat to create a solid object.

Vat polymerization processes are excellent at producing objects with fine details and smooth surface finishes. This makes them ideal for jewelry, low-run injection molding, dental applications, and medical applications such as hearing aids. The main limitation of vat polymerization is the brittleness of the produced objects. For this reason it is not suitable for mechanical parts (lboro.ac.uk).

\section{Stereolithography}

SLA [stereolithography] was one of the world's first 3D printing technologies, invented by Charles Hull in $1984{ }^{3}$ SLA 3D printers use a laser to cure liquid resin into 
hardened plastic.

SLA machines have two main setups: top-down and bottom-up. These refer to the orientation of the laser and the part as it is being printed. Each approach has pros and cons, depending on the use case.

In a top-down setup, the laser source is above the tank and the part is built facing up. The build platform begins at the very top of the resin vat and moves downward after every layer. A top-down machine can handle very large build sizes and is faster than bottom-up machines, but it costs more and requires a specialist to operate. Also keep in mind that changing material in a top-down orientation printer requires emptying the whole tank, which can be time consuming and inefficient.

In a bottom-up machine, the light source comes from beneath the resin tank and the part is built facing upside down. The tank has a transparent bottom with a silicone coating that allows the light of the laser to pass through but prevents the cured resin from sticking to it. After every layer, the cured resin is detached from the bottom of the tank, as the build platform moves upward. This is known as the peeling step. A bottom-up machine is lower cost and more widely available but has a smaller build size and material range than a top-down setup. Bottom-up also requires more post-processing, a result of the extensive use of supports. $^{8}$

The SLA process follows these steps:

1. First, a liquid photopolymer is filled into a vat or tank. 2. A concentrated beam of ultraviolet light or a laser is focused onto the surface of the vat or tank. The beam or laser creates each layer of the desired 3D object using 
cross-linking or by degrading the polymer at a specific location. This step is repeated layer by layer until the 3D object is built to completion.

An SLA object has high resolution and accuracy, clear details, and a smooth surface finish. SLA is quite versatile and can be applied to many different use cases since photopolymer resin formulations have a wide range of optical, mechanical, and thermal properties to match those of standard, engineering, and industrial thermoplastics.

Formlabs (formlabs.com) uses a bottom-up orientation for its 3D printers. It is common for desktop 3D printers to take this approach.

\section{Direct Light Processing}

DLP (direct light processing] is nearly identical to SLA, except it uses a digital light projector screen to flash a single image of each layer all at once. Each layer is composed of square pixels, called voxels, since the projector is a digital screen. In a way, it is similar to an eight-bit ancestor of SLA in the same way that eight-bit drawings have more defined individual square pixels. Since each layer is exposed all at once, DLP can have faster print times compared with SLA, which solidifies a layer in cross sections. $^{4}$

\section{Continuous Direct Light Processing}

CDLP [continuous direct light processing], also known as CLIP [continuous liquid interface production], produces objects in the same way as DLP but relies on the continuous motion of the build plate on the $Z$ axis. This 
results in faster build times because the printer is not required to stop and separate the part from the build plate after each layer is produced.

\section{POWDER BED FUSION}

PBF (powder bed fusion) technologies produce a solid part using a thermal source that induces fusion, sintering, or melting between the particles of a plastic or metal powder one layer at a time. Most PBF technologies have mechanisms for spreading and smoothing thin layers of powder as a part is constructed, resulting in the final component being encapsulated in powder after the build is completed. The most common applications are functional objects, complex ducting (hollow designs), and low-run parts production.

The main variations in PBF technologies come from different energy sources, such as lasers or electron beams, and the powders used in the process, such as plastics or metals. Polymer-based PBF technologies allow for innovation in that there is no need for support structures. This makes creating objects with complex geometries easier.

Both metal and plastic PBF objects typically are strong and stiff, with mechanical properties that are comparable to, or sometimes even better than, the bulk material. There is a range of post-processing methods available that can give objects a very smooth finish. For this reason, PBF is often used to manufacture functional metal parts for applications in the aerospace, automotive, medical, and dental industries.

The limitations of PBF tend to be surface roughness and 
shrinkage or distortion during processing, as well as the challenges that arise from powder handling and disposal. (lboro.ac.uk).

\section{Selective Laser Sintering}

SLS (selective laser sintering] is the most common additive manufacturing technology for industrial applications. The technology originated in the late 1980s at the University of Texas at Austin. ${ }^{6}$ An SLS 3D printer uses a high-powered $\mathrm{CO}_{2}$ laser to fuse small particles of polymer powder. The SLS process follows these steps:

1. First, a bed is filled with powder.

2. The inside of the printer is then heated to near the powder's melting point. This allows the laser to effectively finish what the heat started and sinter, or coalesce, the powdered material to create a solid structure. This step is repeated, layer by layer, until the object is completed.

3. Finally, the object, still encased in loose powder, is cleaned with brushes and pressurized air.

In contrast to SLA and FDM, SLS does not require an object to have support structures. This is because the unfused powder supports the part during printing. This makes SLS ideal for objects with complex geometries, including interior features, undercuts, and negative features.

Parts produced with SLS printing typically have excellent mechanical characteristics, meaning they are very strong. Objects with thin walls cannot be printed because there is a minimum $1 \mathrm{~mm}$ limitation, and thin walls in large models may warp after cooling down.

The most common material for selective laser sintering 
is polyamide (nylon), a popular engineering thermoplastic with great mechanical properties. Nylon is lightweight, strong, and flexible, as well as stable against impact, chemicals, heat, UV light, water, and dirt. Alumide, a blend of gray aluminum powder and polyamide, and rubberlike materials can also be used.

The combination of low cost per part, high productivity, and established materials make SLS a popular choice among engineers for functional prototyping and a costeffective alternative to injection molding for limited-run or bridge manufacturing.

Selective Laser Melting and Direct Metal Laser Sintering Both SLM [selective laser melting] and DMLS (direct metal laser sintering] produce objects via a method similar to SLS. Unlike SLS, however, SLM and DMLS are used in the production of metal parts. SLM fully melts the powder, while DMLS heats the powder to near melting temperatures until it chemically fuses. In practice, SLM and DMLS are functionally the same.

Unlike SLS, SLM and DMLS require support structures to compensate for the high residual stresses generated during the build process. Support structures help to limit the possibility of warping and distortion. DMLS is the most well-established metal additive manufacturing process and has the largest installed base.

\section{Electron Beam Melting}

EBM [electron beam melting] uses a high-energy beam rather than a laser to induce fusion between particles of metal powder. A focused electron beam scans across a 
thin layer of powder, which causes localized melting and solidification over a specific cross-section. An advantage of electron beam systems is that they produce less residual stress in objects, meaning there is less need for support structures. EBM also uses less energy and can produce layers quicker than SLM and DMLS. The minimum feature size, powder particle size, layer thickness, and surface finish, however, are typically lower quality than SLM and DMLS. EBM requires the objects to be produced in a vacuum, and the process can be used only with conductive material. $^{2}$

\section{Multijet Fusion}

MJF (multijet fusion) is essentially a combination of the SLS and material-jetting technologies. A carriage with nozzles, similar to those used in inkjet printers, passes over the print area, depositing a fusing agent on a thin layer of plastic powder. Simultaneously, a detailing agent that inhibits sintering is printed near the edge of the part. A high-power infrared radiation energy source then passes over the build bed and sinters the areas where the fusing agent was dispensed, while leaving the rest of the powder untouched. The process repeats until the object is completed (protolabs.com).

\section{MATERIAL JETTING}

Of all the additive manufacturing processes, material jetting is most comparable to the inkjet printing process. In the same way that an inkjet printer places ink layer by layer onto a piece of paper, material jetting deposits material onto the build surface. The layer is then cured or hardened 
using ultraviolet light. This is repeated layer by layer until the object is completed. Since the material is deposited in drops, the materials are limited to photopolymers, metals, or waxes that cure or harden when exposed to UV light or elevated temperatures.

Material jetting is ideal for realistic prototypes, providing excellent detail, high accuracy, and smooth surface finish. Material jetting allows a designer to use multiple colors and multiple materials in a single run. This makes the process great for low-run injection molds and medical models. It also allows support structures to be printed from a dissolvable material that is easily removed after building. The main drawbacks of material-jetting technologies are the high cost and brittle mechanical properties of the UV-activated photopolymers (lboro.ac.uk).

Nanoparticle Jetting NPJ (nanoparticle jetting) is a process in which a liquid containing metal nanoparticles or support nanoparticles is loaded into the printer via a cartridge. The liquid is then jetted, similar to an inkjet printer, onto a build tray through thousands of nozzles in extremely thin layers of droplets. High temperatures inside the building chamber cause the liquid to evaporate, leaving behind a metal object [additivemanufacturing.media].

\section{Drop-on-Demand}

DOD (drop-on-demand) material jetting printers have two print jets: one to deposit the build material, typically a waxlike liquid; and another for a dissolvable support material. Similar to material extrusion, a DOD printer follows a 
predetermined path and deposits material in a pointwise fashion to build layers of an object. This machine also employs a fly-cutter, a single-point cutting tool, that skims the build area after each layer to ensure a perfectly flat surface before printing the next layer. DOD technology is typically used to produce waxlike patterns for lost-wax casting (used to duplicate a metal sculpture cast from an original sculpture] and mold-making applications. ${ }^{9}$

\section{BINDER JETTING}

A binder jetting process, also referred to as 3DP, uses two materials: a powder and a binder. The binder, which is typically a liquid, acts as the adhesive for the powder. A print head, much like that in an inkjet printer, moves horizontally across the $x$ and $y$ axes to deposit alternating layers of the powder and the binder. The platform holding the bed of powder, which the object is printed on, lowers as each layer is printed. This is repeated until the object is completed. Like SLS, the object does not need support structures since the powder bed acts as support. The powder materials can be either ceramic-based, such as glass or gypsum, or metal, such as stainless steel.

Ceramic-based binder jetting, which uses a ceramic powder as the material, is best for aesthetic applications that need intricate designs such as architectural models, packaging, molds for sand casting, and ergonomic verification. It is not intended for functional prototypes, as the objects created are quite brittle.

Metal binder jetting, which uses a metal powder as the material, is well suited for functional components and more cost effective than SLM or DMLS metal parts. 
The downside, however, is the metal parts have poorer mechanical properties (lboro.ac.uk).

The same people who created binder jetting also created Desktop Metal (desktopmetal.com], a 3D printer system using this technology.

\section{DIRECT ENERGY DEPOSITION}

DED (direct energy deposition) creates objects by melting powder material as it is deposited, similar to material extrusion. It is predominantly used with metal powders or wire and is often referred to as metal deposition since it is exclusive to metals. DED relies on dense support structures, which are not ideal for creating parts from scratch. This makes it best suited for repairing or adding material to existing objects such as turbine blades.

Metal direct energy is what Relativity Space uses to print its rocket parts. Because of the size of the parts it needs to build, it uses a custom machine.

\section{Laser Powder Forming}

Laser powder forming is also known by its proprietary name, LENS [Laser Engineered Net Shaping], developed at Sandia National Labs (sandia.gov). The process uses a deposition head that consists of a laser head, powderdispensing nozzles, and inert gas tubing. The deposition head melts the powder as it is ejected from the nozzles to build an object layer by layer. The laser creates a melt pool on the build area, and powder is sprayed into the pool, where it is melted and then solidified. 


\section{Electron Beam Additive Manufacturing}

EBAM [electron beam additive manufacturing] uses an electron beam to create metal objects by welding together metal powder or wire. In contrast to laser powder forming, which uses a laser, electron beams are more efficient and operate under a vacuum that was originally designed for use in space. ${ }^{5}$

\section{SHEET LAMINATION}

Sheet lamination processes include LOM llaminated object manufacturing] and UAM [ultrasonic additive manufacturing]. You might be familiar with LOM-it is basically the same technology used by the laminator you may have had as a child. To laminate a piece of paper, you place the paper in a laminator pouch composed of two types of plastic: PET (polyethylene terephthalate) on the outer layer and EVA (ethylene-vinyl acetate) on the inner layer. A heated roller then adheres the two sides of the pouch together so the paper is fully encased in plastic. The same basic process is used to build objects (lboro.ac.uk).

UAM, on the other hand, builds metal objects by fusing and stacking metal strips, sheets, or ribbons. The layers are bound together using ultrasonic welding. The process is done on a machine able to CNC (computer numerical control] mill the workpiece as the layers are built. The process requires removal of the unbound metal, often during the welding process. UAM uses metals such as aluminum, copper, stainless steel, and titanium. The process can bond different materials, build at a fast rate, and make large objects practically, while requiring relatively little energy since the metal is not melted. 


\section{THE FUTURE}

The ability to go from a digital file to a physical object rapidly with many different materials allows you to create something you could only imagine in your wildest dreams. Additive manufacturing enables individuals and companies to create without having to involve thirdparty manufacturers, enabling them to go from idea to prototype much faster. Additive manufacturing is even being used to go to space. We have just begun to imagine all the applications for additive manufacturing. Stay tuned to see what people build in the future.

\section{Acknowledgments}

Thank you to Jordan Noone [@thejordannoone] and Michael Fogleman (@FogleBird) for reading an initial draft of this article.

\section{References}

1. Jones, G. 2019. Direct metal laser sintering (DMLS)simply explained. All3DP; https:/lall3dp.com/2/directmetal-laser-sintering-dmls-simply-explainedl.

2. Murr, L. E., Gaytan, S. M. 2014. Advances in additive manufacturing and tooling. In Comprehensive Materials Processing, eds. S. Hashmi, C. J. Van Tyne, G. F. Batalha, and B. Yilbas, 135-161. Elsevier; https://www.sciencedirect. com/topics/chemistrylelectron-beam-melting.

3. Norman, J. Chuck Hull invents stereolithography or 3D printing and produces the first commercial 3D printer. History of Information; https:/lwww. historyofinformation.com/detail.php?id=3864.

4. Redwood, B. Additive manufacturing technologies: an 
overview. 3D Hubs; https:/lwww.3dhubs.com/knowledgebaseladditive-manufacturing-technologies-overviewl.

5. Shuhe, C., Gach, S., Senger, A., Haoyu, Z. 2018. A new $3 \mathrm{D}$ printing method based on non-vacuum electron beam technology. In Journal of Physics: Conference Series 1074:012017; https://www.researchgate.net/ publication/328169730_A_new_3D_printing_method_ based_on_non-vacuum_electron_beam_technology.

6. University of Texas at Austin. 2012. Selective laser sintering, birth of an industry; https:/lwww.me.utexas. edu/news/news/selective-laser-sintering-birth-of-anindustry.

7. V., C. 2019. University of Maine creates the world's largest 3D printed boat. 3D Natives; https:II www.3dnatives.com/en/3d-printed-boat-university-ofmaine-161020195/\#!.

8. Varotsis, A. B. Introduction to SLA 3D printing. 3D Hubs; https:I/www.3dhubs.com/knowledge-baselintroductionsla-3d-printingl.

9. Zhang, L. 2019. Characteristics of drop-on-demand droplet jetting with effect of altered geometry of printhead nozzle. In Sensors and Actuators A: Physical, 298; https:I/www.sciencedirect.com/sciencelarticlelabs/ piilS0924424719312701.

Jessie Frazelle is the cofounder and chief product officer of the Oxide Computer Company. Before that, she worked on various parts of Linux, including containers, and the Go programming language.

Copyright (C) 2020 held by ownerlauthor. Publication rights licensed to ACM. 